\title{
Letter to the Editors on the van Oostrom's Paper
}

\author{
Sung-Won Kim', Dong-Young Noh², Jae-Gahb Park²
}

1Department of Surgery, Seoul National University Bundang Hospital, 300 Gumi-dong, Bundang-gu, Seongnam-si, Gyeonggi-do, 463-707, Korea; ${ }^{2}$ Department of Surgery, Seoul National University College of Medicine, 28 Yongon-dong, Chongno-gu, Seoul, 110-744, Korea

I am very happy to write my opinion regarding Dr. Oostrom's paper.

First of all, I agree with Dr. Oostrom's shortened BRCA counselling protocol. Actually, in my clinical practice, I have already adopted the shortened protocol and usually two sessions are enough for non-mutation carriers. Although my experience in genetic counselling is not that long, I hardly encounter patients with psychological distress that need additional counselling sessions.

There are some reasons for the successful adoption of a shortened BRCA counselling protocol for patients without psychological problems in Korea. First, the incidence of breast cancer in Korea is lower than that of Western countries ( 1 in 40 women will develop breast cancer by the age of 70 years). Therefore, women are less afraid of breast cancer compared to those in Western countries.

Second, most of the BRCA testing is not initiated by the patients but by their doctors. In Korea, the history of the BRCA testing is quite short and hereditary breast cancer is not well recognised by the public. So, most of the counsellees are not aware of the impact of BRCA mutation until the end of their first counselling session. Third, there is no genetic session counselling fee in Korea. Practically, therefore, it is better to adopt a shortened counselling protocol if there are no elevated distress levels and a low prevalence of mental health problems.

The mutation carriers, however, might need psychological support to cope with the breast and ovarian cancer risk for family members and for themselves. Although we can adopt the shortened protocol for non-mutation carriers, we should provide an opportunity for additional counselling sessions for BRCA mutation carriers.

Correspondence to Jae-Gahb Park, MD PhD e-mail: jgpark@plaza.snu.ac.kr 